\title{
OPEN INNOVATION IN MOBILE AND CONVERGENT COMMUNICATIONS
}

\author{
Organizer: José Jiménez \\ Telefónica I+D \\ Spain \\ jimenez@tid.es
}

The concept of open innovation seems to be very well suited to information and communication technologies. In the first place, as in any other field, the complexity of the new technology makes it necessary for companies to use ideas, processes and inventions from other players. As in any other field, we need the collaboration of the university, of SMEs, of inventors, and of the general public.

But open innovation is particularly applicable to ICT.

First of all, telecommunications imply talking and transmitting information to others. This requires using a common language and a communication protocol. This has to be agreed beforehand and it has to be standardized. Agreeing in a common standard requires the effort and collaboration of many and the suggestions cannot come only from one side. This is further complicated when we talk about convergence between mobile and fixed. Ideas from both worlds have to be taken together and combined.

In the second place, ICT is not just telecoms but the result of the collaboration between transmission engineers, radio experts, and information technology scientists. The most known result, the Internet, is not just a telecommunications protocol, but a suite of solutions adapted to the computers and networks.

But this already complex scenario has evolved. The Internet is becoming much more than a protocol, turning into a place where new services and ideas in the areas of information services, communications, and electronic commerce are developing; it is becoming, more a more, a space (the cyberspace) where people meet and interact.

We cannot do things alone.

Unfortunately, the complex nature of the open innovation process does not contribute to making things simple: There are many entry points and not less exit possibilities. The process is complicated for all: telecommunicationes companies, universities, opera-

Please use the following format when citing this chapter:

Jiménez, J., 2008, in IFIP International Federation for Information Processing, Volume 287, Open IT-Based Innovation: Moving Towards Cooperative IT Transfer and Knowledge Diffusion, eds. León, G., Bernardos, A., Casar, J., Kautz, K., and DeGross, J. (Boston: Springer), pp. 543-544. 
tors, government agencies. There is significant controversy about the final outcome and the use of the results. Who will benefit? How can we assure everybody gets their share of the results?

In the end, this open innovation process implies reinventing the company, finding a new business model. The information will not, as in the past, flow only in the top-down direction; it is necessary to learn how firms can utilize communities as complementary assets without having ownership or hierarchical control over them.

But this change is not limited to the company and the technological systems that need to change. The more external innovation is sourced by the firm, the more systems, processes, value, and culture also needs to be transformed. There is an internal resistance to open innovation. Company people are trained to think internally, and this tendency is strengthened by concepts such as core competences and Six Sigma, already entrenched in our organizations. How can we overcome those difficulties? Learning to broaden our views, align with the ecosystem, adapt our company tolerance for risk, and put the focus on learning and not just results, are some of the difficulties we are facing.

As usual, the devil is in the details: What is the degree of customer and supplier integration? How do we set up the innovation clusters? How are we going to handle IPRs and patents? What is the policy toward spin-offs and spin-in? How can we stimulate the people to give their ideas for others to use? The well known poker metaphor introduced by Chesbrough is a good image of the difficulties of playing the game: We have to judge and decide with limited information.

In this session we are very lucky to count on three experts who are going to describe their experiences, from the point of view of an operator, a manufacturer and a university professor who is very much involved with telecommunications research. The three different roles of innovation, as suggested by Chesbrough, funding, generating, and bring innovation to the market, are present.

The first presentation, from Telefónica, describes the new process that will transform Telefónica from a telecommunications provider into a service provider, using talent and ideas both from its internal workforce and from the outside world: The "open Telefónica."

The second will consider how a leading telecom manufacturer, Ericsson, is facing this transformation and how they collaborate in European funded and internal projects with universities and other players.

We close our session with the participation of some other partners from industry who will describe their experiences. 